\title{
Use of Human Lysozyme Transgenic Goat Milk in Cheese Making: Effects on Lactic Acid Bacteria Performance
}

\author{
E. C. Scharfen, ${ }^{\star}$ D. A. Mills, $†$ and E. A. Maga* ${ }^{\star 1}$ \\ *Department of Animal Science, and \\ †Department of Viticulture and Enology, University of California, Davis 95616
}

\begin{abstract}
Genetically engineered goats expressing elevated levels of the antimicrobial enzyme lysozyme in their milk were developed to improve udder health, product shelf life, and consumer well-being. The purpose of this study was to evaluate the effect of lysozyme on the development of lactic acid bacteria (LAB) throughout the cheese-making process. Raw and pasteurized milk from 7 lysozyme transgenic goats and 7 breed-, age-, and parity-matched nontransgenic controls was transformed into cheeses by using industry methods, and their microbiological load was evaluated. The numbers of colony-forming units of $\mathrm{LAB}$ were determined for raw and pasteurized goat milk, whey, and curd at $d 2$ and at $d 6$ or 7 of production. Selective plating media were used to enumerate lactococcal species separately from total LAB. Although differences in the mean number of colony-forming units between transgenic and control samples in raw milk, whey, and cheese curd were nonsignificant for both total LAB and lactococcal species from d 2 of production, a significant decrease was observed in both types of LAB among d 6 transgenic raw milk cheese samples. In pasteurized milk trials, a significant decrease in LAB was observed only in the raw milk of transgenic animals. These results indicate that lysozyme transgenic goat milk is not detrimental to LAB growth during the cheese-making process.
\end{abstract}

Key words: lysozyme, cheese ripening, lactic acid bacteria, transgenic

\section{INTRODUCTION}

Infection caused by the pathogenic invasion of the mammary gland results in reduced milk production, changes in milk composition that lead to decreased yields and altered taste, and the potential loss of the animal, either as the result of culling for chronic masti-

Received December 1, 2006.

Accepted April 25, 2007.

${ }^{1}$ Corresponding author: eamaga@ucdavis.edu tis or death from acute infection. Enhancing the secretion of antimicrobial compounds in milk through the use of genetic engineering has been investigated as an approach to improving udder health and reducing the incidence of mastitis (Wall et al., 2005; Maga et al., 2006a). As a means of increasing the level of naturally produced lysozyme in ruminant milk, a line of genetically engineered dairy goats was created for elevated expression of human lysozyme (HLZ) in their milk (Maga et al., 2003). Increased lysozyme in the milk is expected to improve food safety and prolong shelf life through the inhibition of pathogenic bacteria in addition to providing in vivo protection against mastitis. However, the impact on cheese production of such changes in milk composition must be determined to better evaluate the applicability of this technology to the dairy industry.

The enzyme lysozyme (EC 3.2.1.17) is a naturally occurring antimicrobial protein secreted in the tears, saliva, and milk of all mammals (Masschalck and Michiels, 2003). Lysozyme is a peptidoglycan $N$-acetylmuramoylhydrolase that is primarily effective against gram-positive bacteria. Lysozyme hydrolyzes the peptidoglycan component of the bacterial cell wall and thus lyses the cell (Masschalck and Michiels, 2003). Secretion of lysozyme in milk has evolved as a means of passive immunity transfer between mother and offspring and has been shown to vary with the type of placenta and lactation pattern of the species (Goldman, 2002). In humans, the level of lysozyme produced in the milk increases over the first 4 mo of lactation and is found in concentrations of 100 to $300 \mathrm{mg} / \mathrm{L}$ (Goldman, 2002). Ruminants are comparatively lacking in lysozyme in tears, saliva, and milk (Prieur, 1986). Bovine milk contains $0.13 \mu \mathrm{g}$ of lysozyme $/ \mathrm{mL}$, whereas goats and sheep produce 0.25 and $0.10 \mu \mathrm{g} / \mathrm{mL}$, respectively (Chandan et al., 1968).

Hen egg white lysozyme is used in many foods as an additive against microbial growth and possesses generally recognized as safe (GRAS) standing with the World Health Organization/FDA (Masschalck and Michiels, 2003). Lysozyme is added during the production of cheese to protect against the development of Bacillus 
cereus, a known food pathogen that can also cause offflavors and curdling (López-Pedemonte et al., 2003). Lysozyme has also been used to prohibit late "blowing," the fermentation of lactate by Clostridium tyrobutyricum to form butyric acid, acetic acid, carbon dioxide, and hydrogen during cheese ripening (Bottazzi et al., 1996; Danyluk and Kijowski, 2001; Masschalck and Michiels, 2003).

Although the growth of contaminating pathogenic bacteria is a concern in terms of consumer health and product quality, the ripening and development of the sensorial characteristics of cheese are associated with changes in the lactic acid bacteria $(\mathbf{L A B})$ profile. The amplification of lysozyme expression in milk intended for cheese production therefore poses a potential threat to the ability of the LAB population to proliferate. However, observations of LAB challenged by lysozyme during Grana Parmesan cheese making indicate that the functionality of the LAB population is not impeded (Ottogalli et al., 1983; Neviani et al., 1996). An examination of lysozyme resistance in 39 strains of Lactobacillus spp. and 4 strains of Streptococcus thermophilus commonly found in cheese starter cultures showed that most strains possessed a natural resistance to lysozyme or developed a resistance with repeated exposure, as measured by the levels of carbohydrate fermentation, proteolytic activity, increase in milk viscosity, and acidification (Neviani et al., 1996). In a similar study, growth and acid production were evaluated for 36 strains of LAB isolated from Grana whey starters, which, with the exception of only a few strains of Lactobacillus helveticus, performed normally when in the presence of lysozyme (Ottogalli et al., 1983). Culture media impregnated with various levels of lysozyme confirmed the resistance of a cheese starter culture to concentrations of up to $2,500 \mathrm{U} / \mathrm{mL}$ of lysozyme (Bester and Lombard, 1990). Other work has also indicated that the presence of lysozyme does not have a negative impact on cheese making, because Grana cheeses manufactured with the addition of lysozyme produced a whey with lower titratable acidity than control cheeses but could proliferate in the presence of lysozyme (Grazia et al., 1984).

A line of genetically engineered dairy goats designed to express HLZ in the mammary gland was generated as an approach to improve udder health, resistance to mastitis, and longevity and safety of the milk and milkderived food products (Maga et al., 2006a). Milk from HLZ transgenic goats had an increased shelf life, and in vitro assays demonstrated that HLZ was inhibitory against the growth of Escherichia coli, Staphylococcus aureus, and Pseudomonas fragi but did not affect the growth of Lactococcus lactis (Maga et al., 2006c). In addition, pasteurized milk from transgenic does was capable of modulating gut microbiota when consumed by animal models, indicating a biological effect of active HLZ (Maga et al., 2006b). This study was designed to evaluate how lysozyme affects the bacteria necessary for cheese production by following its effects throughout a traditional goat cheese production method to determine direct applicability to producers. Because HLZ is efficacious against gram-positive bacteria such as LAB, there are concerns about whether cheese making will be adversely affected. Levels of LAB were monitored for raw and pasteurized milk, whey, and cheese curd throughout processing by using milk from HLZ transgenic and nontransgenic controls. Here we demonstrate that the presence of HLZ throughout the cheesemaking process was not detrimental to production.

\section{MATERIALS AND METHODS}

\section{Transgenic Goats and Milk Collection}

The generation of transgenic goats expressing HLZ in the mammary gland under control of the bovine $\alpha_{\mathrm{s} 1}$-CN gene has been described previously (Maga et al., 2003). The line of HLZ transgenic goats used for this study was of Alpine and Toggenburg origin, and the goats expressed active HLZ in their milk at levels of $270 \mu \mathrm{g} / \mathrm{mL}$ (Maga et al., 2006a). Analysis of the milk composition of transgenic animals through regular testing with the DHIA indicated that the fraction of milk yield represented by fat and protein were within the normal variance of nontransgenic control herdmates (Maga et al., 2006a). Control goats were nontransgenic animals from the same herd of origin matched for age, breed (Alpine and Toggenburg), and parity. Cheesemaking trials were conducted for 2 consecutive lactations in the spring and summer of each year. In the first year, milk from 7 individual lactating transgenic females in their first $(n=2)$, second $(n=2)$, third $(n=$ $1)$, fourth $(n=1)$, and fifth $(n=1)$ parity and equal numbers of age-, breed-, parity-, and stage of lactationmatched nontransgenic controls was used. In the second year of trials, a total of 8 does in each group (transgenic and nontransgenic controls) in their first $(n=2)$, second $(n=2)$, third $(n=2)$, fourth $(n=1)$, and fifth $(n=1)$ parity were used. The transgenic and control does in their second, third, fourth, and fifth parities were the same as those used in yr 1 trials. Animals were kept in dry lots and fed the same diet, alfalfa hay during the day with $3.3 \mathrm{~kg}$ of concentrate (corn, oat, barley, cottonseed) offered at each milking. All does were milked by machine in a milking parlor twice a day throughout the length of the lactation. Milk collection for all trials was done at the morning milking. For raw milk trials, milk from each doe was collected individually and weighed. A 2-L volume from each doe 
was taken to make individual cheeses, and the remainder was pooled with the residual from the other does for a given treatment (transgenic or nontransgenic control). For pasteurized milk trials, milk from all animals in a given treatment (transgenic or control) was collected and pooled. A 20-mL representative milk sample was taken from each of the individual milk collections and pooled collections and was frozen for analysis of lysozyme levels and activity as previously described (Maga et al., 2006a). All animals were housed in facilities approved by the Association for Assessment and Accreditation of Laboratory Animal Care.

\section{Cheese Production}

The cheese-making process used was a modified version of that described by Le Jaouen (1987) for the traditional French goat cheese St. Marcelin. In the first year of the study, raw milk cheese was made from each 2-L sample collected from an individual animal. Additionally, $2 \mathrm{~L}$ of milk was taken from each batch of pooled milk to make a composite cheese sample. Immediately after milking, each milk sample was poured through a standard milk filter into individual food-grade plastic buckets, and $0.2 \mathrm{~mL}$ of liquid veal rennet (Dairy Connection Inc., Madison, WI) diluted in $60 \mathrm{~mL}$ of lukewarm water and $0.1 \mathrm{~g}$ of mesophilic starter culture consisting of L. lactis ssp. lactis and L. lactis ssp. cremoris (Ezal, Rhodia Food, Paris, France) were added directly to the individual buckets. The milk was then incubated in the plastic buckets for $24 \mathrm{~h}$ at $25^{\circ} \mathrm{C}\left( \pm 2^{\circ} \mathrm{C}\right)$ until a firm curd had formed. At $24 \mathrm{~h}$, a $20-\mathrm{mL}$ sample of whey was taken from the surface of each batch of cheese for analysis. The remaining whey was then ladled off the curd by hand, and each sample of curd was hand-ladled into $2 \mathrm{St}$. Marcelin plastic cheese molds (Fromagex, Rimouski, Québec, Canada), where the remaining whey was allowed to drain from the curd as the cheeses formed. Thus, 2 cheeses were made from each milk sample. After $24 \mathrm{~h}$ of draining, cheeses were removed from their molds, hand salted, and replaced in the molds for an additional $24 \mathrm{~h}$. Cheeses were then turned out of the molds onto a wire cheese rack with appropriate ventilation and ripened for $4 \mathrm{~d}$ at $20^{\circ} \mathrm{C}$ $\left( \pm 1^{\circ} \mathrm{C}\right)$. Thirty-two cheeses were made for each trial: 14 from the 7 individual HLZ transgenic animals, 14 from the 7 individual nontransgenic controls, 2 from pooled transgenic milk, and 2 from pooled control milk. A total of 3 raw milk trials were conducted from August through September of 2005, with all samples for each trial collected and processed on the same day, for a total of 96 cheeses for analysis.

In the second year of the study, pasteurized milk trials were conducted. A total of $15 \mathrm{~L}$ of milk was col- lected from each of the 2 pools (transgenic and control) and weighed. Milk was pasteurized at $74^{\circ} \mathrm{C}$ for $30 \mathrm{~s}$ (Safgard Pres-Vac Home Pasteurizer, The Schlueter Co., Janesville, WI). Again, a 20-mL representative milk sample was taken before and after pasteurization for lysozyme analysis. After cooling to $27^{\circ} \mathrm{C}$ in a water bath, $0.8 \mathrm{~mL}$ of rennet diluted in $100 \mathrm{~mL}$ of lukewarm water and $0.2 \mathrm{~g}$ of the same mesophilic starter culture was added to the milk. Incubation and cheese making followed the same process as described above for raw milk cheese. In addition, in the second year of trials, the $\mathrm{pH}$ of milk, whey, and curd was monitored, as was the volume of whey expelled from the curd at $24 \mathrm{~h}$. For each trial, a total of 10 cheeses were made for each treatment (transgenic and control). A total of 3 pasteurized milk cheese trials were conducted in May 2006, producing a total of 60 cheeses for analysis. One replicate of a raw milk trial was also conducted concurrently to compare raw and pasteurized results with the same samples, with 5 cheeses produced for each treatment, producing a total of 10 cheeses for analysis.

\section{Enumeration of $L A B$}

Milk (raw and pasteurized prior to starter inoculation), whey, and cheese curd at $\mathrm{d} 2$ and at $\mathrm{d} 6$ (raw milk trials) or 7 (pasteurized milk trials) of production were sampled to determine the numbers of LAB present at each time point. Standard selective media for LAB identification and enumeration were used to enumerate lactococci, streptococci, and enterococci (M17 medium, Oxoid, Basingstoke, UK) from total LAB [de Man, Rogosa, Sharpe (MRS) medium, Oxoid] from each sample (Guerzoni et al., 1999; Mannu et al., 2002; Ercolini et al., 2003; Badis et al., 2004; Öner et al., 2004). Lactose was added to the M17 agar during medium preparation (50 $\mathrm{mL}$ of a $10 \% \mathrm{wt} / \mathrm{vol}$ solution per liter of medium). Prior to plating, samples were first diluted. Milk $(\mathrm{t}=0 \mathrm{~h})$ and whey $(\mathrm{t}=24 \mathrm{~h})$ samples $(100 \mu \mathrm{L}$ each $)$ were diluted to a final concentration of $0.01 \%$ in $0.85 \%$ sterile $\mathrm{NaCl}$ solution. At d 2 and at d 6 or 7 of production, cheese curd $(10 \mathrm{~g})$ from each cheese was collected and prepared according to the methods described by Mannu et al. (2002). Curd was collected by sterile means and homogenized in $90 \mathrm{~mL}$ of sterile $2 \%$ (wt/vol) sodium citrate, then diluted in $0.85 \% \mathrm{NaCl}$ as with the milk and whey. Each diluted sample $(18.5 \mu \mathrm{L})$ was plated in 6 replicates onto M17 and MRS square grid plates (Falcon Integrid, Becton Dickinson Labware, Bedford, MA) by track dilution as described by Jett et al. (1997). Samples were incubated at $37^{\circ} \mathrm{C}$ for $24 \mathrm{~h}$. The total number of colony forming units per lane was counted with ChemiImager 4400 software (Alpha Innotech, San Leandro, CA). 


\section{Statistical Analysis}

The difference between means in the number of colonies formed for transgenic vs. control samples were analyzed by ANOVA (raw milk trials) or Student's $t$-test (pasteurized milk trials; Microsoft Office Excel 2003, Microsoft, Redmond, WA). Values are reported as the mean $\log (\mathrm{cfu} / \mathrm{mL})$ or $\log (\mathrm{cfu} / \mathrm{g}) \pm$ standard deviation.

\section{RESULTS}

\section{pH Evolution}

The $\mathrm{pH}$ of milk, whey, and curd were found to be the same for both HLZ transgenic and nontransgenic control samples. Both raw and pasteurized milks had a pH of 6.8. Similarly, both raw and pasteurized whey had a $\mathrm{pH}$ of 4.25 , whereas raw curd at $\mathrm{d} 2$ had a $\mathrm{pH}$ of 4.0 and pasteurized curd had a $\mathrm{pH}$ of 4.25.

\section{Whey Production}

The amount of whey expelled from the curd at $24 \mathrm{~h}$ was measured for both raw pooled and pasteurized pooled milk cheeses in yr 2 . We found that more syneresis had occurred by $24 \mathrm{~h}$ in the raw milk curds (8- to 15 -fold greater whey volume) than in the pasteurized milk curds. However, there was no difference between HLZ transgenic and nontransgenic control samples.

\section{LAB Profile}

The prevalence of starter LAB (SLAB) and total LAB throughout the cheese-making process was determined with selective plating media for cheeses made with milk from HLZ transgenic and nontransgenic control goats. Bacterial growth on MRS media indicated total LAB numbers [nonstarter LAB (NSLAB) plus SLAB] present, whereas growth on M17 media was taken to indicate numbers of lactococci, streptococci, and enterococci in raw milk samples and numbers of SLAB (Lactococcus spp.) in whey and curd samples, because once the milk is inoculated with the starter culture, growth of Lactococcus spp. will dominate the growth of the other cocci (Guerzoni et al., 1999; Ercolini et al., 2003). In individual raw milk cheese trials, the trend in development of the LAB populations found in both HLZ transgenic and nontransgenic control samples was one of rapid growth from the time of initial milk collection prior to inoculation through sampling of the whey and into the time of collection of the first curd at $d 2$ of production. Levels of total LAB and Lactococcus spp. decreased by the time of the second curd collection at d $6(144 \mathrm{~h})$ of production for both HLZ transgenic samples and con-
Table 1. Effect of human lysozyme transgenic milk on the growth of lactic acid bacteria (LAB) during cheese production with raw milk

\begin{tabular}{llc}
\hline & \multicolumn{2}{c}{ Mean $\log (\mathrm{cfu} / \mathrm{mL})$ or $\log (\mathrm{cfu} / \mathrm{g}) \pm \mathrm{SD}^{1}$} \\
\cline { 2 - 3 } Sample & \multicolumn{1}{c}{ Transgenic (n) } & Control (n) \\
\hline Starter LAB $^{2}$ & & \\
Raw milk & & \\
Whey & $5.27 \pm 0.09(24)$ & $5.32 \pm 0.21(24)$ \\
Curd, d 2 & $5.58 \pm 0.14(24)$ & $5.60 \pm 0.10(24)$ \\
Curd, d 6 & $6.92 \pm 0.18(96)$ & $6.94 \pm 0.13(96)$ \\
Total LAB & $6.54 \pm 0.20(96)^{*}$ & $6.58 \pm 0.31(96)$ \\
Raw milk & & \\
Whey & $5.49 \pm 0.44(24)$ & $5.58 \pm 0.41(24)$ \\
Curd, d 2 & $5.75 \pm 0.64(24)$ & $5.75 \pm 0.77(24)$ \\
Curd, d 6 & $6.94 \pm 0.46(96)$ & $6.88 \pm 0.48(96)$ \\
\hline
\end{tabular}

${ }^{1} \mathrm{cfu} / \mathrm{mL}$ for fluid samples (milk and whey); cfu/g for curd samples.

${ }^{2}$ Enumeration of lactococci, streptococci, and enterococci in raw milk and Lactococcus spp. in whey and curd by growth on M17 medium (Oxoid, Basingstoke, UK).

${ }^{3}$ Counts taken prior to inoculation with starter culture.

${ }^{4}$ Enumeration of total LAB by growth on de Man, Rogosa, Sharpe medium (Oxoid).

*Samples from transgenic goats were different from nontransgenic controls, $P<0.05$; * Samples from transgenic goats were different from nontransgenic controls, $P<0.01$.

trols (Table 1). The profile of SLAB development was similar across all 3 collection periods, increasing over the first $48 \mathrm{~h}$ and decreasing by $144 \mathrm{~h}$. The total LAB profile was more variable across trials.

Initial mean numbers of LAB colony-forming units found in raw milk samples were not significantly different between control and transgenic samples either for lactococci, streptococci, and enterococci $(P=0.219)$ or for total LAB $(P=0.319$; Table 1$)$. When whey samples were evaluated $24 \mathrm{~h}$ later, the numbers of both types of LAB increased in a similar fashion; however, levels of SLAB and total LAB in HLZ transgenic whey samples were not different from nontransgenic controls $(P=$ 0.602 and $P=0.523$, respectively). The numbers of bacterial colonies had further increased by the second day of cheese production in both transgenic and control samples but were not significantly different $(P=0.833$ SLAB; $P=0.054$ total LAB). By the sixth day of production, when the final samples were taken, the LAB load had begun to decline, as demonstrated by the drop in average number of colony-forming units in both the control and transgenic samples (Table 1). Cheese made with milk from transgenic animals had a significantly lower mean number of SLAB $(P=0.025)$ and total LAB $(P=0.006)$ on $\mathrm{d} 6$ of curd collection, suggesting an inhibitory effect of the HLZ transgenic samples.

For pooled pasteurized milk trials, the same trend was observed as with raw milk trials, with the profile of LAB development being one of rapid growth between whey and curd at $\mathrm{d} 2$, followed by a drastic decline in bacterial numbers by d 7 of production for both SLAB 
Table 2. Effect of human lysozyme transgenic milk of the growth of lactic acid bacteria (LAB) during cheese production with pasteurized milk

\begin{tabular}{lcc}
\hline & \multicolumn{2}{c}{ Mean $\log (\mathrm{cfu} / \mathrm{mL})$ or $\log (\mathrm{cfu} / \mathrm{g}) \pm \mathrm{SD}^{1}$} \\
\cline { 2 - 3 } Sample & Transgenic (n) & Control (n) \\
\hline Starter LAB $^{2}$ & $\mathrm{ND}^{4}(18)^{* *}$ & $3.71 \pm 0.84(18)$ \\
Raw milk & $2.39 \pm 2.09(18)$ & $2.54 \pm 2.27(18)$ \\
Pasteurized milk & \\
Whey & $5.56 \pm 0.59(18)$ & $5.67 \pm 0.43(18)$ \\
Curd, d 2 & $7.02 \pm 0.17(30)$ & $7.09 \pm 0.08(30)$ \\
Curd, d 7 & $5.48 \pm 0.18(30)$ & $5.51 \pm 0.08(30)$ \\
Total LAB & $\mathrm{ND}(18)^{*}$ & $2.27 \pm 1.97(18)$ \\
Raw milk & $\mathrm{ND}(18)$ & $1.19 \pm 2.05(18)$ \\
Pasteurized milk & & \\
Whey & $5.81 \pm 0.48(18)$ & $4.09 \pm 3.54(18)$ \\
Curd, d 2 & $7.25 \pm 0.24(30)$ & $7.16 \pm 0.18(30)$ \\
Curd, d 7 & $5.75 \pm 0.03(30)$ & $5.75 \pm 0.10(30)$ \\
\hline
\end{tabular}

${ }^{1} \mathrm{cfu} / \mathrm{mL}$ for fluid samples (milk and whey); cfu/g for curd samples.

${ }^{2}$ Enumeration of lactococci, streptococci, and enterococci in raw and pasteurized milk samples and Lactococcus spp. in whey and curd by growth on M17 medium (Oxoid, Basingstoke, UK).

${ }^{3}$ Counts taken prior to inoculation with starter culture.

${ }^{4} \mathrm{ND}=$ none detected $(<10 \mathrm{cfu} / \mathrm{mL})$.

${ }^{5}$ Enumeration of total LAB by growth on de Man, Rogosa, Sharpe medium (Oxoid).

*Samples from transgenic goats were different from nontransgenic controls, $P<0.05$; **Samples from transgenic goats were different from nontransgenic controls, $P<0.01$.

and total LAB (Table 2). However, in contrast to the raw milk trials, levels of SLAB $(P=0.739)$ and total $\mathrm{LAB}(P=0.780)$ in transgenic and control samples were not significantly different at the final time point $(\mathrm{d} 7$ of production). Similar to the raw milk trials, there were no significant differences between the milk of transgenic and control animals in the levels of SLAB and total LAB at $\mathrm{d} 2$ of production $(P=0.079$ and $P=$ 0.109 , respectively) or in the whey $(P=0.654$ and $P=$ 0.194, respectively). Differences between HLZ transgenic and nontransgenic control samples were also nonsignificant for both SLAB and total LAB in pasteurized samples $(P=0.250$ and $P=0.326$, respectively). In raw milk, HLZ transgenic samples had a significantly lower number of lactococci, streptococci, and enterococci $(P=0.006)$ and total LAB $(P=0.032)$ than control raw milk samples. The one replicate of raw milk trials conducted in the second year followed the same patterns of LAB growth as seen in yr 1 raw milk trials (data not shown). Furthermore, in both yr 1 and yr 2, HLZ levels in milk were consistent, as determined by Western blot (Figure 1).

\section{DISCUSSION}

To better evaluate the applicability of genetic engineering as a technology to ameliorate animal welfare,

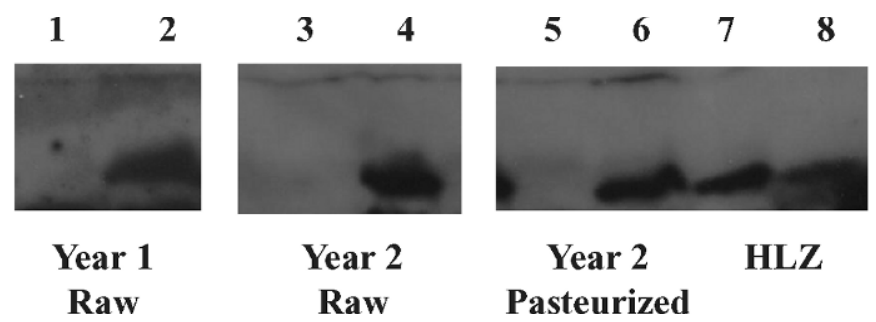

Figure 1. Human lysozyme (HLZ) levels in milk used for cheese making in yr 1 and 2. Milk from each cheese-making trial was subjected to SDS-PAGE with standardized amounts of protein $(30 \mu \mathrm{g})$ and Western blotting with a polyclonal antibody specific to HLZ. Lanes 1 and 3 contain raw pooled milk from nontransgenic control animals in yr 1 and yr 2 trials, respectively; lanes 2 and 4 contain pooled raw milk from HLZ does in yr 1 and 2, respectively; lane 5 contains pooled pasteurized milk from nontransgenic controls in $\mathrm{yr}$ 2 ; lanes 6 and 7 contain raw and pasteurized milk, respectively, from HLZ transgenic does in yr 2; and lane 8 contains the HLZ standard.

product shelf life, and consumer health by producing antimicrobials in the mammary gland, it is important to understand the impact on production processes such as cheese making. One of the most important parameters contributing to the value of cheese is LAB development. The proliferation of LAB is necessary for the acidification of milk, which leads to the formation of curd. Here we report the effect of elevated lysozyme expression on LAB development during cheese production with both raw and pasteurized milk. The presence of mammary-expressed HLZ in the milk of transgenic goats at levels 1,100 times greater than is naturally occurring in goat milk had no obvious adverse effects on cheese making.

Surprisingly, in raw milk trials, the numbers of LAB were not significantly different in transgenic and nontransgenic control samples in the early stages of processing. However, later in the process, by d 6 of production, there was a significant decrease in the mean number of both the Lactococcus genera and total LAB, representing both SLAB and NSLAB. This trend was more consistent among trials for SLAB (Lactococcus spp.). This may be because the culture used to inoculate milk was a combination of $L$. lactis ssp. lactis and $L$. lactis ssp. cremoris, whereas total LAB growth was the result of both deliberate inoculation with starter culture and NSLAB from environmental contamination, and was therefore of unknown origin, quantity, and lysozyme sensitivity.

A diverse array of LAB strains are involved in the acidification and ripening of both raw milk and inoculated milk cheeses. For instance, 725 LAB isolates have been characterized from raw goat milk, including strains from Lactobacillus, Lactococcus, Leuconostoc, Streptococcus, and Pediococcus (Badis et al., 2004). Two 
populations of $\mathrm{LAB}$ play a role in the transformation of milk into cheese. Inoculated SLAB ferment lactose to produce lactic acid. The resulting drop in $\mathrm{pH}$ catalyzes the change of fluid milk into curd. As the SLAB die off, the cells lyse, releasing enzymes into the medium, which break down proteins and fats into peptides, AA, and fatty acids. As the initial SLAB numbers decrease, a second wave of NSLAB begins to develop. These bacteria produce additional breakdown products that contribute further to the complex flavor, texture, and aroma of cheese (Azarnia et al., 2006).

Our results indicate that milk from HLZ transgenic goats does not inhibit the initial bacterial proliferation and acid production necessary for early curd formation during cheese production (as seen in the comparison of $\mathrm{pH}$ values). However, milk from transgenic animals does appear to advance LAB cell lysis in raw milk cheeses. Accelerated cell lysis equates to the release of key proteolytic enzymes from the LAB at an earlier stage for the more rapid production of cheese flavor and aroma. It is possible that this early decrease in LAB levels may have an impact on cheese ripening when raw milk from HLZ transgenic animals is used, although the reduction in SLAB and total LAB growth at $d 6$ of raw milk cheese making was less than 1 log unit, implying that there may be little biological significance. In the one replicate of raw milk cheese making done in yr 2, the pattern of LAB growth in HLZ and control milk followed the same trend as yr 1 trials despite differences in the initial load of LAB. However, because only one replicate was performed, it precludes conducting a valid statistical analysis to determine whether any differences existed between yr 1 and yr 2 raw milk trials.

Various methods have been investigated to reduce the ripening time of cheese. These approaches include the use of attenuated starters, the addition of enzymes, encapsulation of ripening enzymes, elevated ripening temperature, and genetically engineered LAB strains (Azarnia et al., 2006). Attenuated starters have been produced by treatment with lysozyme (Law et al., 1976). Lysozyme-treated cells resulted in a 3-fold increase in the level of free AA, indicating increased proteolysis. It is possible that the advancement of LAB cell lysis seen here when lysozyme produced in milk is used for cheese manufacture can have the same effect on proteolysis. Further studies will be conducted to determine whether the presence of HLZ in milk is directly responsible for the early death of LAB or whether it is associated with the effect because of indirect causes such as influencing the initial load of bacteria.

Pasteurized milk trials were conducted in yr 2 with pasteurized pooled milk from the same groups of goats used in yr 1 trials. In HLZ milk, there were higher levels of SLAB after pasteurization than in the raw
HLZ milk; however, this was not consistent, because it was only seen in 1 of the 3 replicates, perhaps indicating some sort of contamination of the equipment. It should be noted, however, that SLAB levels in postpasteurized HLZ milk were similar to those seen in pasteurized control milk. Thus, the starting bacterial load for cheese making was similar.

In following the progression of LAB growth with pasteurized milk, we found no significant differences between HLZ transgenic and nontransgenic controls for either SLAB or total LAB during cheese making. The levels of LAB found in both our raw milk and pasteurized milk trials were in the range of those reported in the literature for goat milk, with levels of LAB ranging from 2.26 to $6.95 \log (\mathrm{cfu} / \mathrm{mL})$ in raw milk and $<10 \mathrm{cfu} /$ $\mathrm{mL}$ to $3.33 \log (\mathrm{cfu} / \mathrm{mL}$ ) in pasteurized goat milk (Novella-Rodriguez et al., 2004). However, raw HLZ transgenic milk in yr 2 trials did have significantly lower numbers of lactococci, streptococci, and enterococci and total LAB than raw control milk. This difference was not seen in yr 1 trials and could be due to the time of year the trials were conducted (September vs. May) and levels of bacteria in the environment at the time. Quantification of HLZ levels in raw milk used in yr 1 and yr 2 trials confirmed that HLZ levels were not responsible for this difference in initial bacterial load because the HLZ levels were consistent between trials (Figure 1). This result, along with the fact that the nontransgenic controls also had lower levels of initial bacteria in yr 2, indicates an environmental cause for this difference.

In general, the decrease in counts of both SLAB and total LAB at the end of the process (d 2 curd vs. $d 6$ or 7 curd) was greater in pasteurized milk trials (decrease of $1.5 \mathrm{log}$ units) than in raw milk trials (decrease of 0.4 $\log$ units). This result can be attributed to the fact that curd sampling was carried out a day later in the pasteurized trials (d 7) than in the raw milk trials (d 6). Furthermore, the magnitude of this decrease in each year was similar between transgenic samples and their respective controls, with the exception of the growth of total LAB with raw milk. Here, total LAB counts in transgenic samples decreased 0.53 log units from $d 2$ to $d 6$, with the counts of controls decreasing by 0.35 $\log$ units. This result corresponds to our conclusion that levels of total LAB differed most significantly at this time point, and hence had a potential impact on ripening.

Our results demonstrated that after pasteurization, HLZ expressed in the milk of transgenic goats had little effect on the development of LAB. The absence of a decrease in numbers of $\mathrm{LAB}$ at later stages of cheese production in pasteurized trials could be correlated with a decrease in lysozyme activity following the pas- 
teurization process. In-gel activity assays demonstrated that milk from HLZ transgenic animals had an approximate $50 \%$ decrease in lytic activity after pasteurization (data not shown). Therefore, in instances in which raw milk cheeses are the desired product, enhanced expression of lysozyme would be expected to retain full bacteriostatic activity against spoilage organisms. It should be pointed out that because the levels of LAB were not affected when pasteurized milk from transgenic animals was used, the producer could still profit from the potential benefits of HLZ to decrease the chance of bacterial contamination and infection in transgenic goats without altering cheese production.

\section{CONCLUSIONS}

The expression of the antimicrobial lysozyme in the milk of dairy animals has the potential to influence animal health, milk longevity, and cheese production. Here we demonstrated that in vivo-produced lysozyme present at levels of $270 \mu \mathrm{g} / \mathrm{mL}$ in goat's milk was associated with a slight reduction in the number of LAB present late in cheese making with raw milk. Earlier lysis had the potential to speed up the ripening process, thereby reducing the aging time and decreasing the cost to the producer. This effect appeared to be lost when milk was pasteurized at $74^{\circ} \mathrm{C}$ prior to processing, indicating that the lysozyme protein was inactivated at this temperature, so no such benefit would be seen in pasteurized milk processing. Therefore, we concluded that the use of this transgenic technology to protect dairy product shelf life against the development of pathogenic and spoilage organisms did not affect pasteurized cheese production and had the potential to improve the characteristics of raw milk cheese production. Further study will focus on the significance of this effect and the other implications of elevated levels of in vivo lysozyme on cheese production and cheese qualities such as ripening, storage, flavor, texture, and aroma. The results of this work demonstrate that, from a technological standpoint, the presence of mammary-expressed lysozyme in the milk of dairy animals does not preclude its use in the manufacture of cheese.

\section{ACKNOWLEDGMENTS}

We thank James Murray and Lucy Joseph for assisting with the design of LAB culturing techniques, Jan Carlson and the staff of the UC Davis Goat Research Facility for the use of their goats, and Moyra Mauger for her assistance with cheese making.

\section{REFERENCES}

Azarnia, S., N. Robert, and B. Lee. 2006. Biotechnological methods to accelerate Cheddar cheese ripening. Crit. Rev. Biotechnol. $26: 121-143$.
Badis, A., D. Guetarni, B. Moussa Boudjema, D. E. Henni, and M. Kihal. 2004. Identification and technological properties of lactic acid bacteria isolated from raw goat milk of four Algerian races. Food Microbiol. 21:579-588.

Bester, B. H., and S. H. Lombard. 1990. Influence of lysozyme on selected bacteria associated with gouda cheese. J. Food Prot. 53:306-311.

Bottazzi, V., B. Battistotti, A. Rebecchi, and S. Bertuzzi. 1996. Germination of Clostridium spores and the action of lysozyme in Grana cheese. Latte 11:80.

Chandan, R. C., R. M. Parry, and K. M. Shahani. 1968. Lysozyme, lipase, and ribonuclease in milk of various species. J. Dairy Sci. 51:606-607.

Danyluk, B., and J. Kijowski. 2001. Influence of the lysozyme monomer on growth of Clostridium tyrobutyricum. Przemysl-Spozwyczy. 55:16-19.

Ercolini, D., P. J. Hill, and C. E. R. Dodd. 2003. Bacterial community structure and location in Stilton cheese. Appl. Environ. Microbiol. 69:3540-3548.

Goldman, A. S. 2002. Evolution of the mammary gland defense system and the ontogeny of the immune system. J. Mammary Gland Biol. Neoplasia 7:277-289.

Grazia, L., C. Chiavari, G. B. Castagnetti, and G. Losi. 1984. Manufacture of Grana cheese with lysozyme. Acidification of whey and sensitivity of thermophilic lactic acid bacteria. Sci. Tecn. Latt. Cas. 35(Suppl.):384-393.

Guerzoni, M. E., L. Vannini, C. Chaves Lopez, R. Lanciotti, G. Suzzi, and A. Gianotti. 1999. Effect of high pressure homogenization on microbial and chemico-physical characteristics of goat cheese. J. Dairy Sci. 82:851-862.

Jett, B. D., K. L. Hatter, M. M. Huycke, and M. S. Gilmore. 1997. Simplified agar plate method for quantifying viable bacteria. Biotechniques 23:648-650.

Law, B. A., M. J. Castanon, and M. E. Sharpe. 1976. The contribution of starter streptococci to flavor development in Cheddar cheese. J. Dairy Res. 43:301-311.

Le Jaouen, J.-C. 1987. A dictionary of French goat cheeses. Pages 164197 in The Fabrication of Farmstead Goat Cheese. Cheesemakers' Journal, Ashfield, MA.

López-Pedemonte, T. J., A. X. Roig-Sagués, A. J. Trujillo, M. Capellas, and B. Guamis. 2003. Inactivation of spores of Bacillus cereus in cheese by high hydrostatic pressure with the addition of nisin or lysozyme. J. Dairy Sci. 86:3075-3081.

Maga, E. A., J. S. Cullor, W. Smith, G. B. Anderson, and J. D. Murray. 2006c. Human lysozyme expressed in the mammary gland of transgenic dairy goats can inhibit the growth of bacteria that cause mastitis and the cold-spoilage of milk. Foodborne Pathog. Dis. 3:384-392.

Maga, E. A., R. G. Sargent, H. Zeng, S. Pati, D. A. Zarling, S. M. Oppenheim, N. M. B. Collette, A. L. Moyer, J. S. Conrad-Brink, J. D. Rowe, R. H. BonDurant, G. B. Anderson, and J. D. Murray. 2003. Increased efficiency of transgenic livestock production. Trans. Res. 12:485-496.

Maga, E. A., C. F. Shoemaker, J. D. Rowe, R. H. BonDurant, G. B. Anderson, and J. D. Murray. 2006a. Production and processing of milk from transgenic goats expressing human lysozyme in the mammary gland. J. Dairy Sci. 89:518-524.

Maga, E. A., R. L. Walker, G. B. Anderson, and J. D. Murray. 2006b. Consumption of milk from transgenic goats expressing human lysozyme in the mammary gland results in the modulation of intestinal microflora. Trans. Res. 15:515-519.

Mannu, L., G. Riu, R. Comunian, M. C. Fozzi, and M. F. Scintu. 2002. A preliminary study of lactic acid bacteria in whey starter culture and industrial Pecorino Sardo ewes' milk cheese: PCR-identification and evolution during ripening. Int. Dairy J. 12:17-26.

Masschalck, B., and C. W. Michiels. 2003. Antimicrobial properties of lysozyme in relation to foodborne vegetative bacteria. Crit. Rev. Microbiol. 29:191-214. 
Neviani, E., M. Gatti, G. T. Tarelli, and R. Divizia. 1996. Lysozyme resistance of lactic acid bacteria. Latte 3:90-91.

Novella-Rodriguez, S., M. T. Veciana-Nogues, A. X. Roig-Sagues, A. J. Trujillo-Mesa, and M. C. Vidal-Carou. 2004. Evaluation of biogenic amines and microbial counts throughout the ripening of goat cheese from pasteurized and raw milk. J. Dairy Res. 71:245-252.

Öner, Z., O. Sağdiç, and B. Șimşek. 2004. Lactic acid bacteria profiles and tyramine and tryptamine contents of Turkish tulum cheeses. Eur. Food Res. Technol. 219:455-459.
Ottogalli, G., A. Galli, L. Laria, and P. Camaschella. 1983. Effect of lysozyme hydrochloride (Afilact) on lactic acid bacteria in whey starters for Grana cheese. Ind. Latte 19:43-48.

Prieur, D. J. 1986. Tissue specific deficiency of lysozyme in ruminants. Comp. Biochem. Physiol. 85B:349-353.

Wall, R. J., A. M. Powell, M. J. Paape, D. E. Kerr, D. D. Bannerman, V. G. Pursel, K. D. Wells, N. Talbot, and H. W. Hawk. 2005. Genetically enhanced cows resist intramammary Staphylococcus aureus infection. Nat. Biotechnol. 23:445-451. 